\title{
The synchronization of superparamagnetic beads driven by a micro-magnetic ratchet $\dagger$
}

\author{
Lu Gao, $\nleftarrow *$ Norman J. Gottron III,, Lawrence N. Virgin and Benjamin B. Yellen* \\ Received 3rd March 2010, Accepted 26th May 2010 \\ DOI: 10.1039/c003836a
}

\begin{abstract}
We present theoretical, numerical, and experimental analyses on the non-linear dynamic behavior of superparamagnetic beads exposed to a periodic array of micro-magnets and an external rotating field. The agreement between theoretical and experimental results revealed that non-linear magnetic forcing dynamics are responsible for transitions between phase-locked orbits, sub-harmonic orbits, and closed orbits, representing different mobility regimes of colloidal beads. These results suggest that the non-linear behavior can be exploited to construct a novel colloidal separation device that can achieve effectively infinite separation resolution for different types of beads, by exploiting minor differences in their bead's properties. We also identify a unique set of initial conditions, which we denote the "devil's gate" which can be used to expeditiously identify the full range of mobility for a given bead type.
\end{abstract}

\section{Introduction}

The flux of objects through periodic potential energy landscapes under the action of external driving force has implications in a number of physical systems, including atom migration on crystal surfaces, ${ }^{1}$ the motion of charge density packets in solids, ${ }^{2,3}$ the movement of vortices in type II superconductors, ${ }^{4}$ fluid driven movement of colloidal particles through optical and magnetic lattices, ${ }^{5-9}$ as well as various problems in the life sciences and chemical kinetics. ${ }^{10}$ From a physical standpoint, these are fascinating non-linear dynamic systems where small changes in a driving parameter can lead to abrupt changes in the pattern of synchronization of the object relative to the underlying lattice. Arnold's tongues and devil's staircases are names used to describe these different regimes of synchronization. Each step of the "devil's staircase", for example, represents a regime where the object's motion is entrained between two driving frequencies of integer ratios. These systems are both physically intriguing as well have practical interest in applications ranging from magnetoresistance ${ }^{2,3}$ to colloidal separation. ${ }^{6,11}$

In our past work on the motion of superparamagnetic beads above a periodic micro-magnet array driven by an external rotating field, we noticed that nearly "infinite separation resolution" between two different types of colloidal beads could be achieved by adjusting the external driving frequency. Specifically, at certain driving frequencies, one type of colloidal bead translated across the array, but another type was effectively immobilized, rocking back and forth about an equilibrium

Duke University, Department of Mechanical Engineering and Materials Science, Center for Biologically Inspired Materials and Material Systems (CBIMMS),Durham, NC, 27708, USA. E-mail: lu.gao@duke.edu; Fax: +1 919 660-5219; Tel: +1919 660-5342; l.virgin@duke.edu; +1 919 660-8963; +1 919 660-5372; yellen@duke.edu; +1919 660-8963; +1 $919660-8261$

$\dagger$ Electronic supplementary information (ESI) available: 3 video clips (S1-S3) are provided to display the bead's motion at different driving frequencies. See DOI: 10.1039/c003836a

$\$$ Co-first author. position above one magnet. At that time, we were unsure whether this behavior was caused by magnetic forcing dynamics or stick/slip behavior and surface adhesion effects. Recent numerical analysis of this problem in 1-D revealed that our previous experimental results could be explained by the magnetic forcing dynamics. ${ }^{7}$ These simulations predicted a devil's staircase hierarchy of the bead's motion with respect to the driving frequency of the rotating field. At very high frequencies, the bead is in a dynamic regime where its time-averaged velocity is zero and it executes a closed spatially bounded orbit. When the driving frequency is reduced below a critical threshold, the bead abruptly begins to move across the array, executing a subharmonic orbit which is spatially unbounded. As the driving frequency is further reduced, the bead moves from one subharmonic regime to another where its time-averaged velocity transitions between different integer ratios in the process of ascending the devil's staircase. The sub-harmonic orbits persist until the driving frequency is reduced below a critical threshold, in which the bead becomes completely phase-locked with the driving frequency and moves with a time-averaged velocity that is linearly dependent on the driving frequency.

Here we provide experimental evidence that phase-locked, sub-harmonic, and closed orbits can be observed in a system of superparamagnetic beads moving in a traveling wave. We also employ asymptotic methods to develop a theoretical proof on the existence of closed orbits, and we directly identify the initial conditions of all possible closed orbits that can exist in any experimental system. We provide a stability analysis to demonstrate which initial conditions would lead to stable $v s$. unstable orbits. At high frequencies, our theoretical results precisely match the numerical simulations, in which each set of initial conditions corresponds to a closed orbit at a particular driving frequency. At lower frequencies, where the asymptotic assumption breaks down, theory diverges from simulation. However, we discovered that as the frequency was reduced further, the theoretical result and numerical simulation again intersected at a unique set of initial conditions which serve as the transition 
point between the dynamic regimes of sub-harmonic and closed orbits, i.e., the entrance of the devil's staircase hierarchy. For this reason, we denote this set of initial conditions as the "devil's gate". Practically, the devil's gate is the set of initial conditions that can be used as a starting point in a numerical simulation to identify the frequency range for which the bead's motion becomes unbounded (sub-harmonic orbits) or remains spatially bounded and effectively immobilized (closed orbit). This discovery is not only of practical interest in improving the design of future magnetic separation systems, it also may be relevant to other fields which experience synchronization with multiple traveling waves. ${ }^{12,13}$

\section{Theory}

Since our experimental system consists of a square lattice of micro-magnets uniformly magnetized along the same direction, we can approximate the field as that produced by a 2-D array of point poles with duty cycle $\gamma=2 \pi d_{\text {mag }} / d$ representing the ratio of the micro-magnet diameter, $d_{\mathrm{mag}}$, to the lattice period, $d$. Due to the fixed uniform magnetization within the array, the pole sign oscillates along the $x$-direction but retains its sign along the $y$-direction. Thus, we can write the pole distribution as a Fourier expansion:

$$
\lambda\left(\xi_{x}, \xi_{y}\right)=\lambda_{0} \sum_{n=1}^{\infty} \sum_{m=-\infty}^{\infty}\left[\cos \left(n \xi_{x}\right)-\cos \left(n\left(\xi_{x}-\gamma\right)\right)\right] \cos \left(m \xi_{y}\right)
$$

where $\xi_{x}=2 \pi x / d$ represents the dimensionless $x$ (or $y, z$ ) position of the bead center relative to the underlying period, with the origin chosen as one of the positive poles.

The experimental parameter $\lambda_{0}=M_{\mathrm{r}} h / d$ is the effective pole density of the substrate, and is a function of the remnant magnetization, $\vec{M}_{\mathrm{r}}$, and the height of the micro-magnets is $h$. Fig. 1 shows the motion of a superparamagnetic bead undergoing one cycle of the external rotating field in the phase-locked regime.

The field produced by the substrate can be determined through separation of variables and matching the boundary conditions of (1). The resulting field of the micro-magnet array in combination with the external magnetic field $H_{\text {ext }}$ rotating in the $x z$-plane at a driving frequency $\omega$ is given by eqn (2), where $\alpha_{n, m}=\sqrt{n^{2}+m^{2}}$ and $\vec{\xi}=\left(\xi_{x}, \xi_{y}, \xi_{z}\right)$. Expression (2) reduces to a 1-D array of line poles when $m=0$ is the only surviving term along the $y$-direction, leading to the corollary expression described in our previous theoretical work. ${ }^{7}$

The magnetic force on a linearly magnetizable spherical bead is computed using the well known expression for the force on (a)

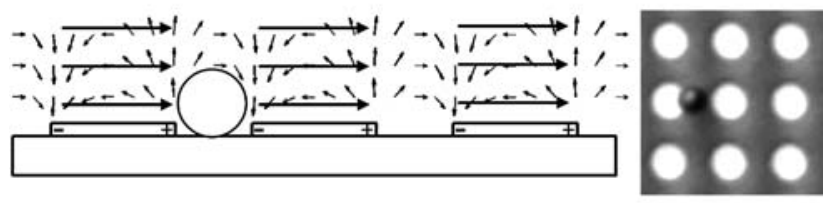

(b)

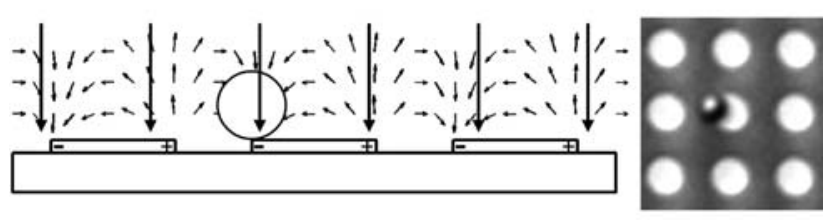

(c)

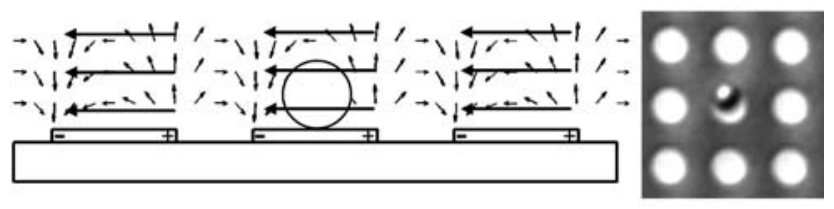

(d)

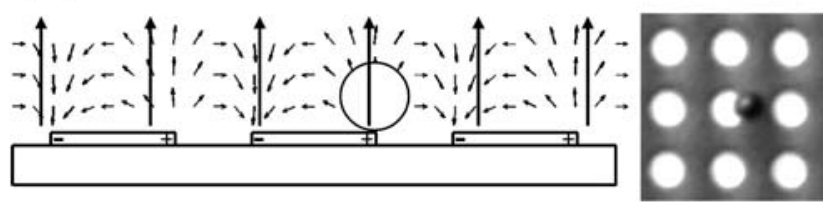

Fig. 1 This figure depicts the phase locked motion of a superparamagnetic bead exposed to a periodic array of micro-magnets and an external rotating magnetic field. The bead is attracted to the region of strongest magnetic field, whose location shifts continually across the substrate as the field completes one revolution of the rotating field (a-d). Corresponding locations in the experimental images are also shown in the right column. In the illustration, the long arrows represent the rotating field while the short ones represent the magnetostatic field.

a point dipole in the presence of a magnetic field gradient, $\vec{F}=\mu_{0}(\vec{m} \cdot \nabla) \vec{H}$, where $\vec{m}=(3 \chi /(\chi+3)) V \vec{H}=\bar{\chi} V \vec{H}$ is the dipole moment of the bead, which is a function of the bead's volume $V$ and the total magnetic field at the bead center, $\bar{H}$. The material property of the bead $\chi$ is accounted for in the effective susceptibility $\bar{\chi}$ that includes corrections for the bead's spherical shape. In order to simplify this problem, we utilize several constraints. First, the bead is assumed to be in contact with the surface at all times (i.e., $\boldsymbol{\xi}_{z}=2 \pi \beta$ where $\beta=$ ald is the ratio of bead radius, $a$, to lattice period, $d$ ), which is a reasonable assumption because the force in the $z$-direction is usually negative. Second, we recognize that the direction of the external field will tend to restore the bead

$$
\vec{H}(\vec{\xi}, t)=H_{\mathrm{ext}}\left[\begin{array}{l}
\sin (\omega t) \\
0 \\
\cos (\omega t)
\end{array}\right]+\lambda_{0}\left[\begin{array}{l}
\sum_{n=1}^{+\infty} \sum_{m=-\infty}^{+\infty} n \cdot \alpha_{n, m}^{-1}\left\{\sin \left(n \xi_{x}\right)-\sin \left(n \xi_{x}-n \gamma\right)\right\} \cos \left(m \xi_{y}\right) \cdot \exp \left(-\alpha_{n, m} \xi_{z}\right) \\
\sum_{n=1}^{+\infty} \sum_{m=-\infty}^{+\infty} m \cdot \alpha_{n, m}^{-1}\left\{\cos \left(n \xi_{x}\right)-\cos \left(n \xi_{x}-n \gamma\right)\right\} \sin \left(m \xi_{y}\right) \cdot \exp \left(-\alpha_{n, m} \xi_{z}\right) \\
\sum_{n=1}^{+\infty} \sum_{m=-\infty}^{+\infty}\left\{\cos \left(n \xi_{x}\right)-\cos \left(n \xi_{x}-n \gamma\right)\right\} \cos \left(m \xi_{y}\right) \cdot \exp \left(-\alpha_{n, m} \xi_{z}\right)
\end{array}\right]
$$


to the $y$-axis, which allows us to set $\xi_{y}=0$. Finally, we consider the case where the external field dominates the substrate's field $\left(H_{\mathrm{ext}}>\lambda_{0}\right)$, leading to the following $x$-force:

$$
\begin{aligned}
F_{x}\left(\xi_{x}, t\right)=F_{0} \sum_{m=-\infty}^{+\infty} \sum_{n=1}^{+\infty} \sum_{l=1}^{2} & \left\{f_{n, m}\left(n \cdot \alpha_{n, m}^{-1}+(-1)^{l}\right)\right. \\
& \left.\cos \left(n \xi_{x}-n \frac{\gamma}{2}+(-1)^{1+l} \omega t\right)\right\}
\end{aligned}
$$

where $F_{0}=\frac{8}{3} \pi^{2} \mu_{0} \bar{\chi} \beta^{3} d^{2} H_{\text {ext }} \lambda_{0}$ and $f_{n, m}=n \times \sin (n \gamma / 2)$ $\exp \left(-2 \pi \alpha_{n, m} \beta\right)$. From (3) it is clear that the forcing terms in the summation represent the superposition of multiple traveling waves of different directions and spatial frequencies.

The bead's velocity as a result of the external force in the overdamped limit (in which inertial terms are ignored) can be written as: $\mathrm{d} \xi_{x} / \mathrm{d} t=F\left(\xi_{x}, t\right) / 6 \pi \eta a$ where the friction coefficient on the particle is assumed to be Stoke's drag on a sphere in a fluid of dynamic viscosity $\eta$. Hydrodynamic effects due to the bead's proximity to the wall are not taken into account; however, this would result merely in multiplication of the friction coefficient by a constant since the bead's vertical position does not change.

Previously, we demonstrated how this equation can be re-cast in the form of a simple non-linear harmonic oscillator for a 1-D line pole distribution when only the first term in the expansion $(n=1)$ is retained, ${ }^{6}$ leading to the result: $\mathrm{d} \varphi / \mathrm{d} \tilde{t}=\sin (\varphi)-\omega / \omega_{\mathrm{C}}$, (dimensionless time $\tilde{t}=t \omega_{\mathrm{c}}$ ), where the critical frequency for the present 2-D system is approximated by:

$$
\omega_{\mathrm{C}}=\frac{4 \mu_{0} \bar{\chi} H_{\mathrm{ext}} \lambda_{0}(2 \pi \beta)^{2} \sin (\gamma / 2)}{9 \eta} \sum_{m=-\infty}^{+\infty} \frac{e^{-2 \pi \sqrt{1+m^{2}} \beta}}{\sqrt{1+m^{2}}}
$$

Assuming only the first term in expression (3) is retained, the phase velocity can be integrated analytically, and after transformation back into real space, the resulting bead velocity is given by:

$$
\left\langle\frac{\mathrm{d} x}{\mathrm{~d} t}\right\rangle=\left\{\begin{array}{ccc}
\omega \frac{d}{2 \pi} & \text { for } & \omega \leq \omega_{\mathrm{c}} \\
\left(\omega-\sqrt{\omega^{2}-\omega_{\mathrm{c}}^{2}}\right) \frac{d}{2 \pi} & \text { for } & \omega>\omega_{\mathrm{c}}
\end{array}\right\}
$$

which demonstrates the bifurcation in the relationship between velocity and frequency. As shown in (5), above the critical frequency $\left(\omega>\omega_{\mathrm{c}}\right)$ when the bead enters the phase-slipping regime, its time-averaged forward progression is positive over all finite driving frequencies. However, when the full expression (3) is numerically simulated both for 1-D line pole and 2-D point pole distributions, sub-harmonic spatially unbounded orbits and closed spatially bound orbits are observed at finite frequencies. ${ }^{7}$

In order to derive an approximate analytical expression and prove the existence of closed spatially bounded orbits, we solve the bead dynamics by using eqn (3) and an asymptotic approach for very high frequency. The solution strategy makes use of the transformation:

$$
\cos \left(n \xi_{x} \pm \omega t-\frac{n \gamma}{2}\right)=\frac{\mathrm{d} \sin \left(n \xi_{x} \pm \omega t-\frac{n \gamma}{2}\right)}{\left(n \dot{\xi}_{x} \pm \omega\right) \mathrm{d} t}
$$

This relationship permits eqn (3) to be integrated analytically for the asymptotic limit, $\omega \gg n \dot{\xi}_{x}$. A bead is considered to be trapped in a spatially bound orbit if it returns to its original position after one half cycle. By using this supposition as the endpoints of the integration, we obtain the following relationship:

$$
0=\sum_{n=1}^{\infty} \sum_{m=-\infty}^{\infty} \sum_{ \pm} f_{n, m}\left(1 \mp n \alpha_{n, m}^{-1}\right) \sin \left(n \xi_{0}-n \frac{\gamma}{2} \pm \delta_{0}\right)
$$

where the coordinate pairs $\left(\xi_{0}=2 \pi x_{0} / d, \delta_{0}=\omega t_{0}\right)$ represent the initial conditions (i.e., position and time) that produce a closed orbit. Conceptually, this is the equilibrium position that the bead visits twice during each complete driving period. By reorganization of (7), we discover a direct relationship between all possible equilibrium positions and initial temporal phases, i.e., a relationship between $\xi_{0}$ and $\delta_{0}$, which allows us to identify all of the possible closed orbits, using the following relationship:

$$
\tan \left(\delta_{0}\right)=\frac{\sum_{m=-\infty}^{+\infty} \sum_{n=1}^{+\infty} n \cdot \alpha_{n, m}^{-1} f_{n, m} \sin \left(n\left(\xi_{0}-\gamma / 2\right)\right)}{\sum_{m=-\infty}^{+\infty} \sum_{n=1}^{+\infty} f_{n, m} \cos \left(n\left(\xi_{0}-\gamma / 2\right)\right)}
$$

The stability of each set of equilibrium orbit locations can be analyzed through a Poincaré cross-section of the bead's trajectory over an integer ratio of a complete driving cycle. In this approach, we assume that the bead is originally displaced from one of the identified initial conditions $\left(\xi_{0}, \delta_{0}\right)$ by a small amount, say $\xi_{x}=\xi_{0}+\varepsilon$, where $\varepsilon$ is a small perturbation. By making use of the identity eqn (6) and integrating over one half cycle, we can determine the trajectory of the perturbation over each cycle which is governed by the differential equation:

$$
\frac{\mathrm{d} \varepsilon}{\mathrm{d} t}=\varepsilon \omega_{0} \Lambda
$$

where $\Lambda$ is given by:

$$
\begin{aligned}
\Lambda=-\omega_{0} \sum_{m=-\infty}^{+\infty} \sum_{n=1}^{+\infty} \sum_{l=1}^{2} & \left\{n \cdot f_{n, m}\left(n \cdot \alpha_{n, m}^{-1}+(-1)^{l}\right)\right. \\
& \left.\cos \left(n \xi_{0}-n \frac{\gamma}{2}+(-1)^{l+1} \delta_{0}\right)\right\}
\end{aligned}
$$

Eqn (9) has the general solution:

$$
\varepsilon(N)=C \exp (\Lambda \times N)
$$

where $N$ is the number of half driving cycles. When $\Lambda$ is negative, the orbit is stable, otherwise it is unstable. See Discussion section.

\section{Experimental}

Our experimental system used an array of $4.9 \mu \mathrm{m}$ circular micromagnets spaced with $8 \mu \mathrm{m}$ periodicity on a square lattice, which was produced by the conventional photolithographic lift-off technique described elsewhere. ${ }^{6}$ The thickness of the magnets was maintained at $\sim 50 \mathrm{~nm}$, and the magnitude of the rotating field was $H_{\text {ext }}=20$ Oe. The experimental system consisted of mixing the magnetic beads at a sufficiently dilute ratio and placing in a fluid cell of $100 \mu \mathrm{m}$ thickness and total area of $1 \mathrm{~cm}^{2}$. The external field was applied using an apparatus reported previously. ${ }^{6}$ The trajectories of superparamagnetic beads with a nominal diameter of $2.7 \mu \mathrm{m}$ (M-270 Dynabeads, Invitrogen), corresponding to a value of $\beta=0.17$, were tracked using a Leica 

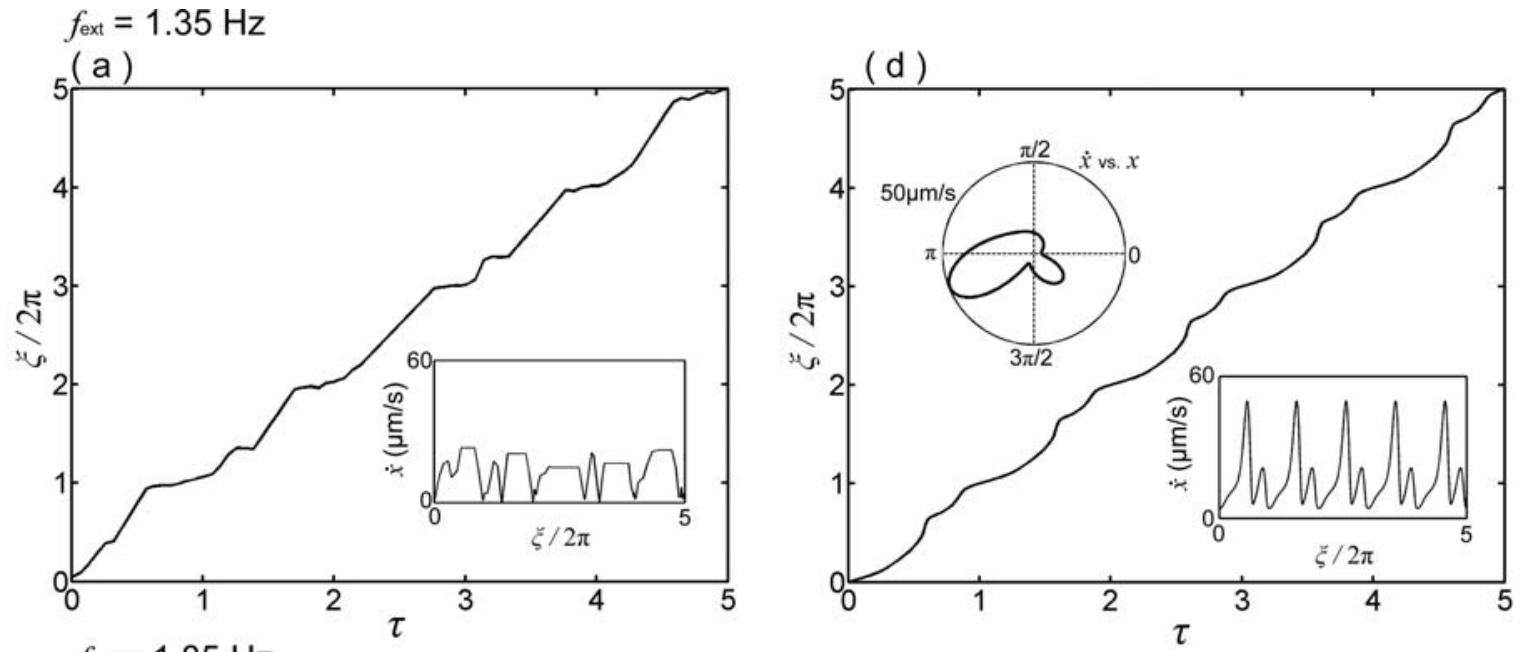

$f_{\text {ext }}=1.85 \mathrm{~Hz}$
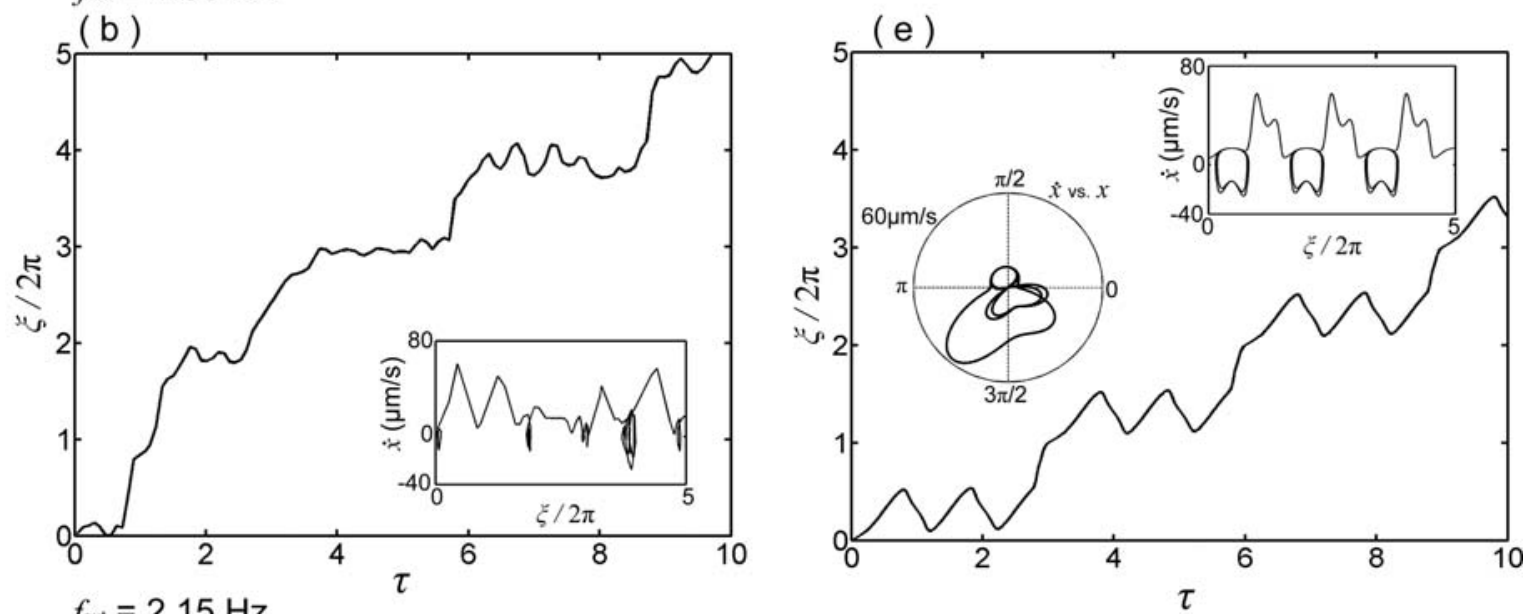

$f_{\text {ext }}=2.15 \mathrm{~Hz}$
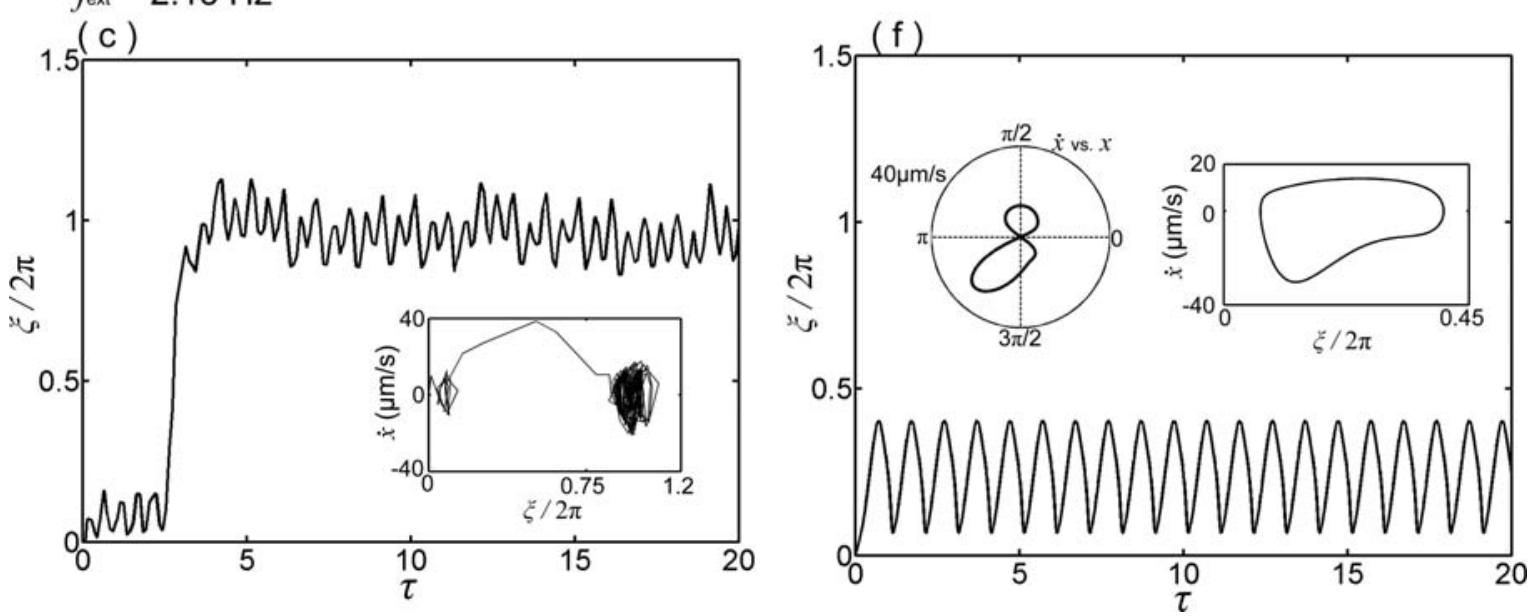

Fig. 2 This figure depicts the experimental (a-c) and numerically simulated (d-f) trajectories of the beads as a function of the number of cycles, $\tau=t f_{\text {ext }}$, of the driving frequency, $f_{\text {ext }}$. A bead driven by a frequency of $1.35 \mathrm{~Hz}$ moves in the phase locked state, where $\langle V\rangle=1$ (a and d). At a driving frequency of $1.85 \mathrm{~Hz}$, the bead's motion is in a sub-harmonic locking state, characterized by superimposed rocking motion and periodic escapes from one magnet to the next, shown in (b and e). At this frequency, the bead's motion is extremely sensitive to the driving frequency, which makes it difficult to precisely control the velocity in the sub-harmonic range since any small fluctuation in the magnetization of the micro-magnets can dramatically change the subharmonic orbit. We present a numerically simulated velocity of $\langle V\rangle=1 / 3$ in order to capture the main features of the experimental trajectory. At a driving frequency of $2.15 \mathrm{~Hz}$, the bead is in a closed orbit state (c and f). Experimental and numerical phase portraits (velocity $v$ s. position) are embedded in each of the figures. In the numerical simulations, we also include a phase portrait in polar coordinates to clearly display the periodicity of the motion. Due to the low sampling rate of our camera, we were unable to capture the fine details of motion. To deal with this issue, the experimental positions were averaged over the nearest two time points, in effect acting as a smoothing filter, in which case the gross experimental behaviour matched simulations reasonably well. 
DM microscope and Simple PCI video tracking software. The experimental trajectories for the driving frequencies of $1.35 \mathrm{~Hz}$, $1.85 \mathrm{~Hz}$, and $2.15 \mathrm{~Hz}$ are provided in Fig. $2 \mathrm{a}-\mathrm{c}$, along with the experimental phase portraits, which depict the velocity $v s$. position. Clearly, the bead is in a phase-locked mode at $1.35 \mathrm{~Hz}$, moving at a normalized velocity of $\langle V\rangle=1$, which is the speed of the first harmonic of the traveling wave (see Video S1 $\dagger$ ). For frequencies slightly above $1.85 \mathrm{~Hz}$ the bead is observed to undergo sub-harmonic phase-locking, where it alternates between rocking and traveling motion in a periodic fashion. Due to slight variation in the magnetization of the micro-magnets along with thermal fluctuations, the orbit is not perfectly subharmonic as is evident in Fig. 2b. For example, the bead moves a distance of one magnet for each of the first two cycles, after which it becomes trapped periodically for several cycles when moving past the next three magnets. In this case, the time-averaged velocity is $\langle V\rangle=1 / 2$ (see Video $\mathrm{S} 2 \dagger$ ). At $2.15 \mathrm{~Hz}$ and above, the bead exhibits pure rocking motion about a single magnet in a closed orbit. Due to thermal fluctuations, the bead occasionally escapes out of the closed orbit, as can be seen in Fig. 2c; however, when it arrives at the next magnet it re-enters the closed orbit and rarely escapes (see Video $\mathrm{S} 3 \dagger$ ).

\section{Results and discussion}

We adjusted our simulation parameters to match the experimental results in Fig. 2a-c. The corresponding trajectories for each frequency are provided in Fig. $2 \mathrm{~d}-\mathrm{f}$, along with a phase portrait plotted in both the Cartesian and polar coordinate system. The polar plot in particular shows that the orbits are indeed closed. All simulations were performed in FORTRAN. In our simulations, we assumed the bead susceptibility and fluid viscosity were: $\bar{\chi}=0.16$ and $\eta=0.011$ Poise in order to reflect the experimental system parameters. Clearly, the simulated trajectories and phase portraits are found to be very similar to experimental observations, providing further evidence that both the sub-harmonic and closed orbits result from magnetic forcing terms as opposed to stick/slip motion with respect to the substrate. The parameter $\lambda_{0}=2.58$ Oe produces the best fit

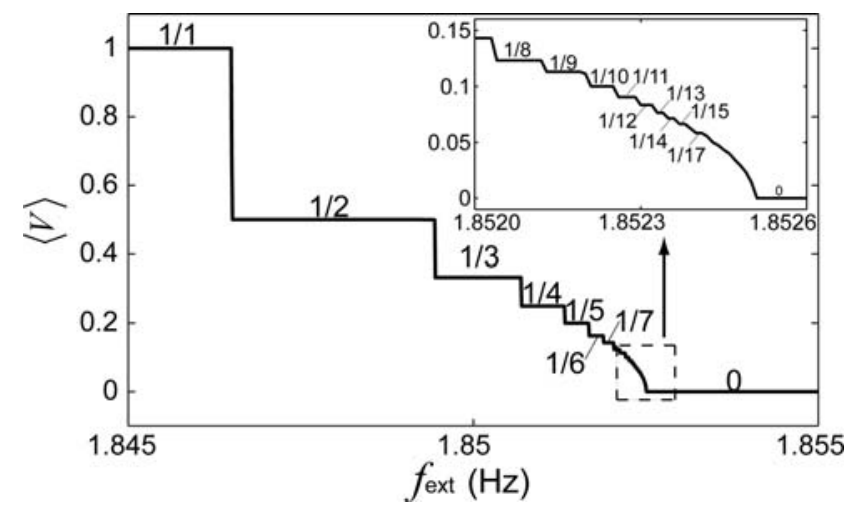

Fig. 3 For $\beta=0.17$, the normalized average velocity, $\langle V\rangle$, of the bead drops from 1 to 0 by increasing the driving frequency only $0.01 \mathrm{~Hz}$. Within this tiny increment, the velocity undergoes the devil's staircase hierarchy from $1 / 1$ to $1 / \infty$, which is extremely sensitive to the driving frequency. between theory and experiment, corresponding to a value of $M_{\mathrm{r}}=413 \mathrm{Oe}$, which is a reasonable value for substrate magnetization but lower than the saturation magnetization of Cobalt. The discrepancy may be due to a slightly higher friction coefficient in the experiments when the beads are close to the surface, or due to slightly non-uniform magnetization of each micromagnet. An example of the normalized velocity vs. frequency distribution is provided in Fig. 3, which is a typical devil's staircase hierarchy. The normalization procedure consists of taking the ratio of the numerically computed velocity of the bead to the equivalent velocity of the driving frequency. When the

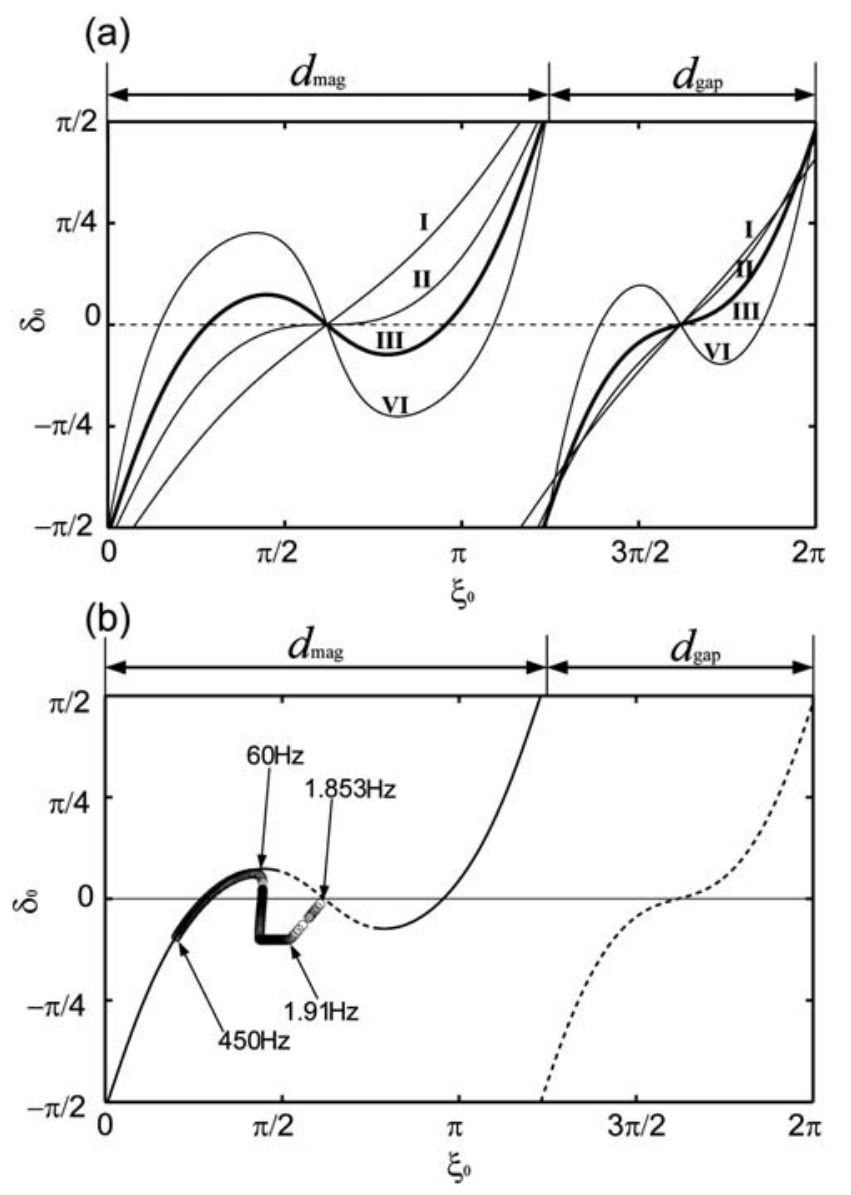

Fig. 4 (a) Theoretical curves (I-IV) derived from eqn (8) depict the initial conditions $\left(\delta_{0} v\right.$ s. $\left.\xi_{0}\right)$ which produce a closed orbit for different values of $\beta$ ranging from (I) $\beta=0.40$, (II) $\beta=0.24$, (III) $\beta=0.17$, and (IV) $\beta=0.10$. Closed orbits were only found in simulations for $\beta$ which has extrema. In case I, the curve is monotonically increasing and the bead never enters a spatially bound closed orbit. Case II demonstrates the boundary where closed orbits can be observed. Case III depicts the experimental conditions and case IV demonstrates the situation for an even smaller bead. The simulated data and all theoretical curves converge at the set of initial conditions we denote the "devil's gate", which is the lowest frequency that the bead can remain in a closed orbit. (b) A stability analysis is provided for case III (i.e., $\beta=0.17$ ). The solid line denotes the stable branch of the oscillation, and the dash line denotes the unstable branch. The numerically identified positions of the closed orbits in the frequency range from 1.853 to $450 \mathrm{~Hz}$ (the data points are denoted by circles " $\bigcirc$ ") provide a good match with the stability analysis. The lowest frequency of $1.853 \mathrm{~Hz}$ has a closed orbit very close to the devil's gate. 
normalized velocity is 1 , the bead is in the phase-locked regime, which occurs below $1.846 \mathrm{~Hz}$. As the driving frequency is further increased, the normalized velocity of the bead becomes entrained in integer ratios of $1 / 2,1 / 3$, and so on, representing different plateaus in the devil's staircase hierarchy. Above a critical driving frequency, the bead's velocity becomes zero as it enters a stable closed orbit, occurring at approximately $1.852 \mathrm{~Hz}$.

In Fig. $4 a$, we show the initial conditions $\left(\delta_{0}, \xi_{0}\right)$ that would produce closed orbits derived from eqn (8) as a function of different bead sizes (i.e., different $\beta$ ). Two characteristic regimes are observed. A monotonically increasing relationship is observed for $\beta>0.24$ (Case I), which is representative for a large bead whose diameter is larger than the micro-magnets. For $\beta<$ 0.24 (Cases II-IV), several extrema begin to emerge and the peaks grow with decreasing $\beta$. In our numerical simulations, we discovered that closed orbits were only possible when extrema in eqn (8) were present.

A stability analysis is provided in Fig. 4b along with the numerically identified closed orbits. The initial conditions of the closed orbit were identified by simulating the bead's motion for up to 10000 cycles. After the orbit became stable, we determined the mean position of the orbit along with the time point when the bead reached its mean position. The results indicate that in the frequency range of $60-450 \mathrm{~Hz}$, the beads approach different points on the stable branch of the stability diagram. In the high frequency regime, the equilibrium position of the closed orbit is on top of the micro-magnet towards one of the poles. As the frequency is lowered to $60 \mathrm{~Hz}$, the location of the stable closed

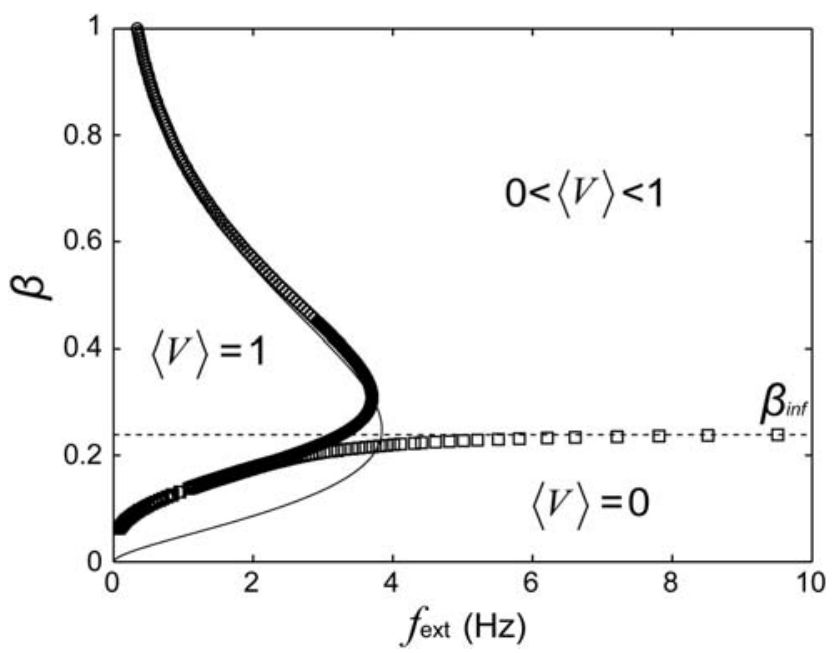

Fig. 5 The mean velocity is plotted as a function of $\beta$ and the external driving frequency, $f_{\text {ext }}$. For low driving frequencies and for $\beta>0.06$ the beads are entrained with the moving potential with time-averaged velocity, $\langle V\rangle=1$. Numerically identified critical frequencies are denoted by circles $(\bigcirc)$. The boundary of this regime is well predicted by eqn (4) for large $\beta$; however, this approximation breaks down when $\beta<0.30$. Closed orbits where $\langle V\rangle=0$ are found only for $\beta<0.24$, where the transition between these two dynamic states is depicted by the boxes $(\square)$. These points were identified using the "devil's gate" as the initial conditions and the procedure outlined in the main text. In the remaining regions of the phase space (i.e. at large frequencies and for $\beta>0.24$ ), the bead can never reach a closed orbit and the time averaged velocity is always between 0 and 1 . orbit shifts towards the middle of the magnet. A second regime is noted below $60 \mathrm{~Hz}$, in which the theoretical and numerically identified closed orbits diverge, most likely due to the reduced validity of our assumption $\omega \gg n \dot{\xi}_{x}$. Numerical simulations suggest the location of the closed orbit continues to move towards the middle of the magnet as the frequency is further reduced. At even lower frequencies, we discovered that simulations again approach the theoretical curve of eqn (8) at the location of the unstable branch, which is at the center of the micro-magnet. Conceptually, this position may be unstable because it is the location of weakest magnetic field and thus weakest potential energy.

The lowest frequency that the bead could remain in a closed orbit occurred at the intersection of theory and simulation data at the position we call the devil's gate. This same inflection point occurs for all bead sizes (Fig. 4a), thus it seems to be a general initial condition that can serve to identify the transition between closed and sub-harmonic orbits, i.e., the onset of the devil's staircase hierarchy commonly observed in non-linear dynamic systems of interacting driven oscillators. We denote this set of initial conditions the devil's gate, because if these initial conditions were used as the starting point of a simulation for any bead size, then a frequency scan can identify the threshold for which the bead transitions into sub-harmonic motion, i.e., enters the devil's staircase hierarchy. The simulation procedure for identifying this frequency threshold is very simple and consists of simulating the bead's motion over one cycle of the driving frequency. The characteristic stopping frequency is identified by the condition in which the ending position of the bead after one cycle changes from moving in the negative direction (back to the location of other closed orbits) to a positive direction where the bead escapes to the next magnet. This theoretical finding significantly simplifies the analysis of this non-linear dynamical system as it allows for the full frequency range of closed orbits to be expeditiously identified.

Another interesting aspect of these simulations is that closed orbits exist only when $\beta$ is below a critical size range of $\beta<0.24$, where the extrema begin to emerge in Fig. 4. A phase diagram was constructed for the bead velocity as a function of $\beta$ in order to identify the regimes where the bead's velocity is phase-locked $(\langle V\rangle=1)$, phase slipping but with open orbit $(0<\langle V\rangle<1)$, or phase slipping in a closed orbit $(\langle V\rangle=0)$. These results are provided in Fig. 5, along with the predicted values of $\omega_{\mathrm{c}}$ from eqn (4). The critical frequency can be theoretically predicted for large values of $\beta$; however, when $\beta<0.30$, theory and simulation diverge. The maximum $\beta$ for which beads become trapped in a closed orbit is also plotted as the line $\beta_{\text {inf }}$.

From an inspection of Fig. 5, it is clear that the separation resolution between different types of beads will be most efficient when $0.06<\beta<0.20$. At these frequencies, the transition between phase-locked and closed orbits is very sharp, i.e., the bead will transition between an open phase-locked orbit $(\langle V\rangle=$ 1) and a closed spatially bounded orbit $(\langle V\rangle=0)$ with very small modulation of the driving frequency. Thus, future magnetic separation systems should focus in this parameter range to most efficiently sort colloidal objects based on their size, charge, mass, or biological activity.

While the mathematics indicates that closed orbits can exist in these systems, it does not provide physical intuition for how or 
why the bead can actually achieve a closed orbit. The transport of objects by periodic ratchets has been studied extensively by many authors and various physical arguments have been made for how the shape of a finite traveling potential can affect an object's velocity over a long time average. Some prior approaches consider the asymmetry in the potential energy landscape and determine the different time periods that an object resides on one or another side of an inflection point in the potential landscape. If conditions are met such that the object returns to its original position within one driving period, then it is assumed that a closed orbit can exist.

We have analyzed the potential energy surface of the bead as it is moving in the traveling wave, and our simulations suggest that this is exactly what happens during one driving cycle. If the bead does not reach a critical location above the micro-magnets within a given fraction of a driving period, then it is forced onto the backside of the wave, retarding its forward progress. For subharmonic motion, the bead never completely makes it back to its original position, and thus there is a net forward advancement of the bead over each driving cycle. These small advances add up over time until a critical position is breached and the bead can advance to the next magnet. The number of cycles it takes for the bead to advance by one magnet is represented by the different plateaus in the devil's staircase. For closed orbits, on the other hand, the bead can make it back to its original position after one driving cycle, in which case there is zero forward advancement and zero time averaged velocity.

We speculate that the reason this is possible in our experimental system is that the pole density of the substrate is fixed and serves as an attractor for the beads. The external rotating field is trying to drive the beads away from a given fixed point to a neighboring fixed point upstream. If the driving frequency is too fast, or the magnitude of the driving field is too small, the bead will not have enough time to make it past the bifurcation and will remain in the neighborhood of a fixed point. Though this may not be the most satisfying intuitive explanation, it explains the gross experimental behavior. The mathematical description provided here, on the other hand, is more quantitatively satisfying because it allows us to identify not only at what frequencies the closed orbits occur, but also the actual trajectory of a closed orbit as well as their dependence on the bead size and the pole distribution of the substrate. These results will be useful in improving future magnetic separation systems based on this non-linear approach.

\section{Conclusion}

We note several key conclusions from this work. First, we show experimentally and theoretically that the superposition of multiple traveling waves can constructively interfere such that the bead becomes entrained in a spatially bounded closed orbit. We show through a combination of theory, simulation, and experiments, that these closed orbits are a result of magnetic forcing dynamics rather than stick/slip motion and adhesion to the substrate. Secondly, we derived an analytical model which predicts the full range of closed orbits that exist for each bead size, and we identified a general set of initial conditions which we denote the "devil's gate" which can be used to predict the onset of the devil's staircase hierarchy. We also provide a stability analysis that demonstrates which initial conditions are stable $v$. unstable. Thirdly, we constructed a phase diagram that shows the range of bead sizes that will be most efficient at achieving infinite separation resolution (i.e., beads that quickly transition between phase-locked motion and closed orbits with small change in frequency). These findings have relevance in the development of future magnetic separation systems that can achieve infinite separation resolution between beads of different sizes (i.e., systems can be tuned to allow beads of one size to move across the array, while beads of another size remain trapped in closed orbits). These results are scalable to different micro-magnet sizes and thus can be used to increase control of colloidal beads in microfluidic systems. Our methodology for determining transition points and identifying unique sets of initial conditions, which defines transition zones between different non-linear dynamical regimes, may also be of relevance to other fields, ranging from transport through crystalline lattices to other dynamical systems that experience synchronization among large numbers of coupled oscillators.

\section{References}

1 O. Pierre-Louis and M. I. Haftel, Phys. Rev. Lett., 2001, 87, 048701. 2 J. Wiersig and K.-H. Ahn, Phys. Rev. Lett., 2001, 87, 026803.

3 S. E. Brown, G. Mozurkewich and G. Grüner, Phys. Rev. Lett., 1984, 52, 2277.

4 C. Reichhardt and F. Nori, Phys. Rev. Lett., 1999, 82, 414.

5 A. Gopinathan and D. G. Grier, Phys. Rev. Lett., 2004, 92, 130602-130604.

6 B. B. Yellen, R. M. Erb, H. S. Son, R. Hewlin, Jr, H. Shang and G. U. Lee, Lab Chip, 2007, 7, 1681-1688.

7 B. B. Yellen and L. N. Virgin, Phys. Rev. E: Stat., Nonlinear, Soft Matter Phys., 2009, 80, 011402-011406.

8 A. Soba, P. Tierno, T. M. Fischer and F. Sagues, Phys. Rev. E: Stat., Nonlinear, Soft Matter Phys., 2008, 77, 060401.

9 P. Tierno, S. V. Reddy, J. Yuan, T. H. Johansen and T. M. Fischer, J. Phys. Chem. B, 2007, 111, 13479-13482.

10 S. H. Strogatz, Nonlinear Dynamics and Chaos: With Applications to Physics, Biology, Chemistry and Engineering, Westview Press, 1994.

11 P. T. Korda, M. B. Taylor and D. G. Grier, Phys. Rev. Lett., 2002, 89, 128301.

12 D. Cubero, J. Casado-Pascual, A. Alvarez, M. Morillo and P. Hanggi, Acta Phys. Pol., B, 2006, 37, 1467-1477.

13 S. I. Denisov, T. V. Lyutyy, E. S. Denisova, P. Hänggi and H. Kantz, Phys. Rev. E: Stat., Nonlinear, Soft Matter Phys., 2009, 79, 051102. 